\title{
DE ESCUELA EVANGELIZADORA COLONIAL A SISTEMA EDUCATIVO COMPETITIVO Y SEGMENTADO EN ISLA DE PASCUA
}

\author{
FROM EVANGELIZING COLONIAL SCHOOL TO A COMPETITIVE AND \\ SEGMENTED EDUCATIONAL SYSTEM ON EASTER ISLAND
}

\author{
Javier Corvalán $R^{1}$
}

\begin{abstract}
Se analiza el desarrollo histórico de la escolarización en Isla de Pascua estableciendo que desde 1917 en ella se siguieron procesos educativos que tuvieron características coloniales, evangelizadoras y occidentalizadoras. En los últimos años y particularmente en la década pasada, en Isla de Pascua se forma un sistema escolar de tipo competitivo que genera diferentes tipos de segmentaciones, en particular socioeconómica y educativa (logros de aprendizaje) con elementos étnicos eventualmente incorporados en las anteriores.

Palabras claves: Isla de Pascua, educación, segmentación socioeducativa.
\end{abstract}

The historical development of the schooling system on Easter Island is analysed, concluding that since 1917 this process has been characterized by having colonial, religious and westernizing features. In the past years and particularly during the last decade, a competitive schooling system has been established on Easter Island. This competitive system has resulted in different kinds of segmentation, especially socio-economic and educational (i.e., learning achievements) with ethnical elements occasionally incorporated.

Key words: Easter Island, education, socio-educational segmentation.

\section{Marco Conceptual, Contextualización y Preguntas Orientadoras}

Como parte del ejercicio creador y recreador constante del Estado-nación las políticas educativas tienen la capacidad de viajar e instalarse en lugares apartados de los países, intentado modelar las sociedades locales en un esquema de organización institucional mayor sin el cual, se asume, la organización político-administrativa y la identidad nacional se desdibujarían. Con esto, los Estados modernos son productores simbólicos (y con ello también asimiladores culturales) tanto como redistribuidores de recursos, generando a nivel local no solo las instituciones con las que sus referentes centrales son vehiculados e instalados en las conciencias de sus ciudadanos, sino también los discursos legitimadores de los procesos mediante los cuales tal organización institucional se lleva a cabo. En particular la escuela, en su versión nacionalrepublicana, ha sido la concreción más evidente del proyecto institucional de Estado-nación en las sociedades modernas. Mediante ella, se crea y se recrea constantemente la comunidad imaginada (Anderson 2007). Si bien la escuela es capaz de generar esta producción simbólica, los contenidos de esta última dependen de las particularidades escolares de cada sociedad y de las dinámicas que la organización institucional de las escuelas establezca en el medio que las circunda. Así por ejemplo, son distintos los procesos que estableció la escuela de los inicios de la escolarización moderna (Archer 1984:60-64) que, como imagen, refiere a una institución casi monopólica, amparada por el Estado o por la Iglesia y a la cual asistían los niños que habitaban en sus inmediaciones, en comparación a las dinámicas sociales establecidas entre escuela, sociedad y comunidad en los tiempos actuales. La funciones prioritarias de aquella escuela fueron las de enseñar las primeras letras (Rojas 2010:58-59), instruir al pueblo (Amunátegui y Amunátegui 1856:81-82) y/o civilizar al indígena (González 2002), es decir, aquello que Durkheim sintetizó como socializar a individuos no necesariamente modernos para desempeñarse en sociedades modernas, complejas y enmarcadas en un Estado-nación (Durkheim 1993).

1 Centro de Investigación y Desarrollo de la Educación (CIDE), Facultad de Educación, Universidad Alberto Hurtado, Santiago, Chile.jcorvala@cide.cl 
En Chile lo anterior, además, es un lento proceso que comienza con la independencia y que adquiere una notoria aceleración en la segunda mitad del siglo XIX, resaltando en este proceso la Ley de Instrucción Primaria de 1860 y la posterior consolidación de la escolarización amparada por el Estado ya entrado el siglo XX, con la Ley de Educación Primaria Obligatoria, de 1920. Con esta última, el país comienza a consagrar un sistema educativo propiamente tal, con preeminencia del Estado como proveedor del servicio pero a la vez con presencia de colegios privados con mayor o menor relación financiera e institucional con aquel (Corvalán et al. 2010). El paradigma central de desarrollo educativo que se afianza en un paso siguiente, en especial con la reforma educacional de 1965, es el de centralismo estatal, buscando la masificación y en lo posible la universalización de la asistencia a la escuela (PIIE 1984). Así, la política educacional desarrolla a partir de la reforma de ese año un importante referente común en un currículo nacional altamente homogéneo (PIIE 1984). El objetivo educativo central de ese momento es la incorporación de todos los niños y adolescentes del país a los códigos básicos de una modernidad que se supone homogénea, intrínsecamente positiva y amparada bajo el Estado-nación. Sobre la base de este conjunto de principios y procesos de la educación entre los siglos XIX y XX se instala, afianza y desarrolla también y de manera acelerada, la escolarización en Isla de Pascua.

Los procesos antes mencionados hacen que la escuela chilena se expanda durante el siglo XX y que se consolide como acción estatal en lugares remotos y apartados del país incorporando a la chilenidad no solo al conjunto de la población marginal, sino también a los pueblos originarios del territorio (Ponce de León Atria 2010). La Isla de Pascua por su lejanía y fuerte etnicidad ${ }^{1}$ es uno de los ejemplos más claros de este proceso de construcción de nacionalidad moderna y de asimilación cultural mediante la educación escolar, la que atraviesa diferentes etapas, inicialmente como una escuela bajo tutela militar, luego una escuela religiosa que se convirtió en una escuela fiscal y posteriormente en una municipal, la que al poco tiempo debió convivir y competir con escuelas privadas. Así, en Isla de Pascua, la historia educacional del siglo XX ha sido la condensación acelerada -en ocho décadas- de procesos que en el resto de Chile comenzaron hace al menos 150 años. Estos cambios educacionales no solo chilenizaron a la población local, aun cuando la reivindicación identitario-cultural está aún presente en la población rapa nui (Gómez 2010), sino que también convivieron con otros cambios socioculturales más amplios en la población nativa los que, particularmente a partir de la década de 1960 y en alguna medida desde antes, estuvieron marcados por instituciones y procesos tan diversos como la Iglesia católica y su monopolio evangelizador (incluyendo la educación), la llegada de la radio y la televisión y el impacto de la globalización y de las relaciones de mercado (Porteous 1981). Todos estos fenómenos cambiaron con fuerza a la sociedad rapa nui de las últimas décadas (McCall 1996), estratificándola de acuerdo con procesos inherentes a las sociedades capitalistas, formando grupos sociales con distintos tipos de capitales materiales y simbólicos y a la vez haciendo que tales capitales y la tendencia de los individuos a conservarlos e incrementarlos impacten en sus expectativas, referentes y decisiones educativas. Considerando lo anterior, las preguntas que orientan la continuidad de este artículo son dos: (i) ¿Cuáles han sido los grandes hitos de la escolarización en Isla de Pascua y cómo ellos reflejan la imposición de procesos y estructuras educativas por parte del Estado chileno? y (ii) ¿Qué antecedentes existen respecto del impacto que ha tenido la formación del sistema escolar en la década de 2000, con la llegada de la educación privada y la competencia por alumnos en la isla?

\section{Metodología}

La metodología utilizada para responder a las preguntas del artículo fue de tres tipos. En primer lugar se recopiló información bibliográfica sobre el desarrollo histórico de la escolarización en la isla. En segundo lugar se analizó información secundaria, principalmente de las bases de datos del Ministerio de Educación sobre el comportamiento de la matrícula escolar en la isla entre los años 2001 y 2010 y las diferencias actuales de las pruebas $\mathrm{SIMCE}^{2}$ en los colegios isleños eligiéndose ese período, ya que es la etapa en la cual la educación escolar en la isla se complejiza con la llegada de colegios privados. Finalmente, se hizo un trabajo de terreno en tres estadías en la isla entre 2010 y 2012 en las cuales se entrevistó a personal de la municipalidad encargado del sector educación, a directores de los colegios y apoderados. En la última estadía en terreno se aplicó una encuesta 
a todos los alumnos de enseñanza media de la isla presentes en ese momento ${ }^{3}$ recogiendo datos relevantes para entender los cambios ocurridos en ese sistema educativo. Por motivos de espacio en este artículo hacemos solo referencias marginales a las entrevistas realizadas y concentramos el análisis en la información histórica y de datos primarios y secundarios de tipo cuantitativo.

\section{Resultados}

\section{Creando chilenos en Polinesia: recuento histórico de la escolarización en Isla de Pascua}

La historia reciente de Isla de Pascua -aquella que se desarrolla después de su descubrimiento por los occidentales en 1722- es, a lo menos, traumática. Después de su avistamiento por marinos holandeses a comienzos del siglo XVIII y durante el resto de ese siglo y el siguiente, la población local quedó a la deriva de numerosos occidentales que pasaron por sus costas, desde exploradores que transportaron moais como souvenir, hasta esclavistas que mataron y encarcelaron sin piedad (Ramírez 2011). Una inflexión en esta historia y que marca además los inicios del primer proceso de escolarización occidental en la isla aun cuando sea de tipo informal es la que realizan los misioneros franceses de la Congregación de los Sagrados Corazones durante la década de 1860 y cuya figura más destacada es Eugenio Eyraud. Esta acción evangelizadora implica una incipiente alfabetización tanto en lengua tahitiana como en francés e incluso con elementos de latín (Edwards 1918:31-32; Annales de la Propagation de la Foi 1867:257). Según la información disponible (Annales de la Propagation de la Foi 1869:323324) tal proceso abarcó a una cantidad importante de niños y adultos de la isla, y se interrumpe a fines de la década mencionada y en la antesala de la anexión a Chile. Existen diversas versiones del interés y el contexto en el cual se produjo esta anexión, en 1888: colonialismo ${ }^{4}$, interés geopolítico $^{5}$ y simple acción expansionista no mayormente reflexionada ${ }^{6}$. Lo cierto es que entre la fecha de anexión y la década de 1960 la isla fue una mezcla de cárcel semiabierta y explotación agrícola privada bajo la custodia del gobierno de Chile (Soto y Fuentes 2010; Stambuck 2010) 7 .

La relación de la isla con Chile se puede dividir en varias etapas, siendo la década recién mencionada (1960) la que marca el inicio de un proceso de modernización insular (McCall 1996; Ramírez 2011). En las etapas iniciales, la isla es un enclave privado y los rapa nui son, en la práctica, ignorados en tanto portadores de derechos (Soto y Fuentes 2010; Stambuck 2010). En este panorama y poco antes de la instalación formal de lo que sería la primera escuela oficial en la isla, en 1917, se dio una suerte de experimento educativo, en 1914, relatado por la investigadora inglesa Katherine Routledge, presente en la isla en ese momento:

\section{La Corbeta Baquedano ha traído algunas novedades... Entre los recién llegados se encontraba un maestro de escuela chileno; él fue enviado por el gobierno y trajo una costosa escuela. La escuela se inició rápidamente, pero después de unos pocos días los niños dejaron de asistir, el maestro declaró que él no era un 'oficial de asistencia' y, desde entonces hasta que abandonamos la Isla, casi un año después, no existió escuela (Routledge 1919:150-151).}

Como dijimos, la primera escuela oficial en la isla es instalada en 1917 lo que ocurre mediante la ley 3.220 que además autoriza la construcción de un lazareto. En ese primer intento educativo formal:

\begin{abstract}
Es al Sub-Delegado Marítimo a quien le corresponde vigilar el funcionamiento de ésta y hacer cumplir la asistencia obligatoria de todos los niños... entre 7 y 14 años de edad. Entre sus obligaciones, está la de enviar a buscar a los niños inasistentes y la de aplicar la pena de 1 a 6 horas de trabajo al padre que se niega a enviar a los niños a la Escuela... sin perjuicio de los castigos escolares correspondientes (Cristino et al. 1984:15).
\end{abstract}

Hacia la década de 1930, observa también Cristino, "la escuela cuenta con 120 alumnos y la enseñanza es impartida irregularmente por las esposas de los Subdelegados de turno" (Cristino et al. 1984:46). Esta primera etapa educativa es una experiencia que de manera irregular comprendía una escolarización de tres años y con un marcado énfasis colonial, occidentalizador y nacionalista como se infiere de las materias enseñadas: 
1. Lecciones de cosas. Por medio de objetos reales de uso ordinario se enseñan los nombres y su pronunciación... 2. Lenguaje... frases sencillas-- género y número... Cambiar las personas, números y tiempos de verbos... 3. Lectura... El orden del silabario... que los niños comprendan el sentido de las palabras... 4. Escritura. Formar en el pizarrón primero... los rasgos fundamentales, las letras, palabras y frases como indica el silabario. 5. Aritmética. Ejercicios de contar... Problemas de adiciones y sustracciones con objetos reales... 6. Todos los días... cantar himnos religiosos, patrióticos y escolares al comenzar y concluir las lecciones. Pasos gimnásticos, bailes escolares... 7. Economía, higiene y labores. Aseo... de las ropas, útiles y habitaciones; lavado y cocina. Costura. Principios de higiene ... 8. Historia y geografía generales y patria. Extensión. Población. Riqueza. Hermosura de Chile. Batallas. Trabajos de las ciudades y los campos. 9. Religión. Doctrina Cristiana. Mandamientos, principales oraciones. Vida de N.S. Jesucristo Lectura por la maestra y después repetición con preguntas, por los alumnos (Cristino et al. 1984:15).

El estudio citado en el párrafo anterior contiene también prolijas observaciones sobre el estado de la educación en la isla en esta primera mitad del siglo XX:

La escuela funcionó desde 1918 en adelante en forma más o menos irregular, dependiendo exclusivamente del Sub-delegado... La enseñanza en la escuela duraba tres años, los cuales... eran suficientes para aprender todo cuanto debían saber. El Sub-delegado enseñaba también los bailes de moda en Chile continental. La escasez de prendas de vestir y la dificultad para conseguirlas cuando arribaba algún barco, hicieron que la enseñanza del bordado y la costura fueran un éxito En 1931, el Comandante don Luis Alvarez Jaramillo... hizo presente la necesidad de atender a la educación de los pascuenses enviando profesores continentales que enseñaran el idioma patrio. Señaló... la falta de recursos... y de atención médica (Alvarez 1931) En 1933, otro capitán de navío, reitera lo señalando... la necesidad del funcionamiento de una escuela pública con maestros chilenos (Cristino et al. 1984:17).

Una visión también crítica de la educación en la isla en ese período se señala en el mismo texto:

En 1934, el doctor Israel Drapkin, médico chileno miembro de la expedición francobelga que visitaba la Isla, señalaba en su informe lo siguiente: 'La instrucción pública en la Isla, hasta hace un año era muy rudimentaria. Largos períodos sin cursos de ninguna especie se alternaban con algunos meses de clases...Sólo... en 1934 se organizó la Escuela Pública, siempre bajo la Dirección Provincial de Instrucción Pública de Valparaíso. Este es el motivo principal de la existencia de una gran cantidad de analfabetos, a pesar de ser los pascuenses muy inteligentes y de tener verdadero afán por los conocimientos de toda índole'. Más adelante agregaba. 'Siendo la Isla de Pascua posesión chilena, el idioma oficial es el español, pero como hasta la fecha la influencia directa de nuestro Supremo Gobierno se ha reducido sólo a la presencia en la Isla del Sub-delegado no es de extrañarse que si bien la mayoría de los varones pascuenses comprende el castellano, sólo un grupo, más bien reducido de ellos, pueda expresarse correctamente en ese idioma'. Finalmente, señalaba que: 'Considerando como analfabetos a todos aquellos nativos que no leen y escriben ningún idioma (una cantidad apreciable de ellos leen y escriben pascuense y tahitiano), y sin contar los niños menores de 10 años, hay actualmente en la Isla 147 analfabetos $(53,89 \%)$, leen y escriben 126 $(46,11 \%)$. Hay 183 niños menores de 10 años' (Cristino et al. 1984:18).

El mismo estudio continúa señalando que:

Hasta 1938 no hubo mayor preocupación por la Escuela y la calidad de la enseñanza en Isla de Pascua, contando con solamente un 
profesor continental. Las esposas de los Subdelegados estaban a cargo de la enseñanza y la escuela era en esa época la única fuente de conocimiento del idioma español. De ahí que su funcionamiento irregular afectaba directamente el aprendizaje de éste. El Gobierno chileno intentó arreglar el local escolar mediante una cláusula estipulada en el contrato de arrendamiento de la Isla a la Compañía Explotadora de Isla de Pascua, la cual no se cumplió (Cristino et al. 1984:19).

Precisamente, en 1938, la educación en la isla es confiada a la Congregación de las Hermanas Misioneras Catequistas del Divino Corazón de Jesús (como consecuencia de que la isla, el año anterior, había pasado a depender eclesiásticamente del Vicariato de La Araucanía), que se hacen cargo de la escuela hasta 1956 (Cristino 2010:46). En ese período la escolarización es ampliada hasta sexto año de preparatorias (cuarto año de primaria desde 1939; quinto desde 1948 y sexto desde 1953) y algunos relatos de algunos rapa nui que vivieron este proceso hacen referencia al clima autoritario de la escuela: "algunos de los testimonios que hacen sobre esta materia los isleños que dejaron sus estudios durante su niñez, ya sea para dedicarse a trabajar o porque no les agradaban las monjas, quienes los maltrataban" (Soto y Fuentes 2010:264). Zurob, por su parte, señala que: "estas religiosas traían consigo los duros métodos pedagógicos de su época, de modo que no dudaban en aplicar el castigo físico en el intento de 'instruir' a los alumnos polinesios" (Zurob 2009a:97) . $^{2}$

A partir de 1956 y como producto de la expansión educativa que se estaba produciendo en el país, la escuela de dependencia netamente fiscal llega a la isla reemplazando al menos parcialmente a la escuela religiosa, dado que algunas hermanas de la congregación siguieron en ella. Esta escuela fiscal completó la oferta de educación básica, de ocho años, en 1968, lo que puede ser visto como una consecuencia de la política nacional que en ese período (específicamente a partir de la reforma educativa de 1965), buscaba la universalización de la educación primaria. Junto con la educación ya en manos de la red oficial del Estado chileno, la década de 1960 significa un proceso de integración paulatina de la población isleña a las estructuras institucionales de la sociedad chilena (Cristino et al. 1984; McCall
1996). En esta etapa se afianza la presencia civil chilena, hasta entonces muy escasa, representada por los funcionarios públicos y sus familias la que sumada a las mayores expectativas educacionales de la población local, genera crecientes demandas sobre la oferta escolar y hace que esta tenga en la misma sala clase de alumnos isleños y continentales. De hecho, Fisher (2005) señala que la remodelación de la nueva escuela y su pleno régimen dentro del sistema escolar chileno en la década de 1960 se debió a la influencia de los chilenos continentales, cada vez más numerosos que pedían una educación propiamente chilena para sus hijos (Fisher 2005:222).

Si bien es cierto en este período se produce un masivo aumento de los niveles de la escolarización de la población isleña más joven (Cristino et al. 1984), hay consenso en que con ello se intensifica también un proceso de transculturación reflejado, por ejemplo, en la pérdida paulatina de la lengua nativa en la comunicación cotidiana (Makihara 2005a, 2005b). Al mismo tiempo, el aumento de las expectativas educacionales que se expresan en la motivación por realizar estudios secundarios y la inexistencia hasta los años noventa de tal nivel completo dentro de la oferta en la isla (ampliada hasta segundo medio en 1976) hace que numerosos jóvenes isleños durante décadas hayan migrado al continente para terminar su escolarización ${ }^{9}$, residiendo la mayoría en hogares estudiantiles (Zurob 2009b). Finalmente, en 1987, la oferta de educación en la isla se amplía, hasta completar la educación media.

\section{La situación reciente de la escolarización en Isla de Pascua}

En la década de 1980 la educación en la isla recibe una de las transformaciones recientes más importantes del sistema escolar chileno: el proceso de municipalización. Con ello, su único establecimiento escolar pasa a depender de la municipalidad local, la que había sido creada en los años sesenta ${ }^{10}$ como parte del proceso de modernización y desarrollo de la isla ${ }^{11}$. Al decir de quienes vivieron este proceso de municipalización escolar, con él la situación educativa no cambió mayormente y eso se debe, probablemente, a que parte de los principios de la regulación educativa propuesta con la reforma educativa de los años ochenta en el país, eran inoficiosos en la isla. En efecto, tales reformas proponen incrementar la competencia entre 
escuelas a fin de mejorar la calidad de la enseñanza (Corvalán et al. 2010), lo que es incoherente en una situación de oferta escolar monopólica y, además, en condiciones de aislamiento. En términos de historia educativa en la isla más importante que el proceso de municipalización de la enseñanza fue el aumento de la oferta escolar de la escuela a la totalidad de la enseñanza media o secundaria durante la década de 1980, lo que disminuyó la migración hacia el continente de parte de adolescentes isleños, generó un aumento de los niveles educacionales de la población local y con ello, a futuro, la demanda por ingreso a la educación superior ${ }^{12}$. Con la creación de dos colegios privados en la década de 2000, que se suman a la oferta municipal ya existente y de acuerdo con la regulación educacional imperante en el país, la educación en Isla de Pascua pasa entonces a organizarse bajo la forma de un sistema educacional de tipo competitivo. A su vez, el colegio municipal se divide en dos establecimientos, uno que conserva su nombre original (Colegio Lorenzo Baeza) y que está limitado a la enseñanza preescolar y básica y otro de enseñanza media (Liceo Aldea Educativa) y que, para tal efecto, construye nuevas instalaciones (UNESCO-MINEDUC-Ilustre Municipalidad de Isla de Pascua 2001). La implementación de un programa de inmersión en lengua rapa nui, a inicios de la década de 2000 , puede ser visto como una de las últimas etapas del desarrollo educativo en la isla. Se trata de una experiencia de la escuela municipal que desde el nivel preescolar hasta el cuarto año básico organiza una de sus secciones bajo esta modalidad y que a partir de las entrevistas realizadas se argumenta que habría impactado tanto en la recuperación lingüística como en los motivos para optar por ese o por otros establecimientos por parte de las familias. Cabe señalar también que los colegios privados de la isla mantienen también una asignatura de lengua rapa nui obligatoria para todos sus alumnos.

\section{El Sistema Educativo actual en Isla de Pascua}

Como ya mencionamos, en la década de 2000 se forma en Isla de Pascua un sistema educativo propiamente tal, compuesto hasta la actualidad, por cuatro establecimientos escolares:

(i) Colegio Lorenzo Baeza (CLB): Se trata del nivel de enseñanza preescolar y básica del antiguo colegio fiscal y municipal de la isla y por lo tanto durante décadas fue la única escuela en el territorio.
Esto hace que la toda población escolarizada de la isla y actualmente mayor de 30 años de edad haya pasado por este colegio lo que hasta el día de hoy genera un fuerte lazo identitario con él (de acuerdo con algunas de las entrevistas realizadas para esta investigación). Por lo mismo, este es todavía el colegio de primera opción para una parte importante de la población rapa nui y según sus autoridades actuales, cerca de un $80 \%$ de sus alumnos pertenecen a este grupo étnico. Se trata actualmente de una escuela de 429 alumnos al año 2010 siendo el establecimiento educacional de mayor tamaño en la isla, concentrando a un $33 \%$ de la matrícula escolar total insular. Una particularidad de este colegio es que, como ya señalamos, desde inicios de la década pasada y desde el nivel preescolar hasta cuarto básico se divide en dos secciones, una de las cuales imparte todas sus materias en lengua rapa nui. Los rendimientos educativos de este colegio, medidos por la prueba SIMCE, son notoriamente menores que los restantes establecimientos como queda expresado en la Tabla 1 . Al mismo tiempo, el año 2011 un total de 119 alumnos (28\% de su matrícula) de este colegio fueron clasificados como "prioritarios" 13 según los criterios del Ministerio de Educación, es decir, de una condición socioeconómica que requiere una atención educativa más costosa y la mayor parte de sus apoderados están clasificados en un nivel socioeconómico "medio", similares al colegio particular subvencionado laico, pero inferior al colegio confesional, referido a continuación. (ii) Colegio Hermano Eugenio Eyraud (CHEE): Se trata de un colegio particular subvencionado fundado por una organización eclesial católica en el año 2005. Es un establecimiento con nivel educacional básico y medio y con 407 alumnos al año 2010, concentrando actualmente a un $31,5 \%$ de los alumnos de la isla. Dos características resaltan respecto de este colegio, su carácter privado-confesional (que lo hace ser identificado como el colegio "católico") y el hecho de tener financiamiento compartido (cobro de mensualidad por escolarización, además de recibir subvención estatal por alumno). Con ello, pasa a ser el único colegio en la isla que cobra por la escolarización siendo este cobro, el año 2012, de $\$ 40.000$ mensuales (U\$D 80). En cuanto a su rendimiento SIMCE, este colegio concentra los puntajes más altos de la isla en todos los niveles educacionales evaluados, tal como se observa en la Tabla 1. De acuerdo con la información del Ministerio de Educación, los 
Tabla 1. Puntajes obtenidos en la prueba SIMCE 2010 en segundo medio por los colegios de Isla de Pascua. 2010 SIMCE test scores achieved by the $10^{\text {th }}$ grades of schools on Easter Island.

\begin{tabular}{|c|c|c|c|}
\hline Establecimiento & Prueba & Promedio & Comparación con $\mathrm{NSE}^{1}$ \\
\hline \multirow{2}{*}{ Liceo Aldea Educativa Rapa nui } & Lenguaje & 237 & $\begin{array}{c}\text { Más bajo } \\
(-22)\end{array}$ \\
\hline & Matemáticas & 240 & $\begin{array}{c}\text { Más bajo } \\
(-16)\end{array}$ \\
\hline \multirow{2}{*}{ Colegio San Sebastián de Akivi } & Lenguaje & 253 & $\begin{array}{c}\text { Similar } \\
(-6)\end{array}$ \\
\hline & Matemáticas & 257 & $\begin{array}{l}\text { Similar } \\
(+1)\end{array}$ \\
\hline \multirow{2}{*}{ Colegio Hermano Eugenio Eyraud } & Lenguaje & 266 & $\begin{array}{l}\text { Más alto } \\
\qquad(+7)\end{array}$ \\
\hline & Matemáticas & 246 & $\begin{array}{c}\text { Más bajo } \\
(-10)\end{array}$ \\
\hline
\end{tabular}

Fuente: elaboración propia a partir de www.simce.cl

${ }^{1}$ Comparación con establecimientos con similar nivel socioeconómico (NSE) en Chile.

apoderados de este colegio pertenecen al nivel socioeconómico medio-alto ${ }^{14} \mathrm{y}$ tienen un promedio de 14 años de escolaridad. No registra presencia de niños categorizados como prioritarios dado que no participa en la política gubernamental de recursos suplementarios para ellos (subvención escolar preferencial). (iii) Colegio San Sebastián de Akivi (CSSA): Fue el primer colegio privado establecido en la isla el año 2003. Se trata de un establecimiento laico y gratuito con un $16 \%$ de alumnos prioritarios, con una clasificación de nivel socioeconómico de rango medio de los apoderados (de acuerdo con la asignación que realiza el Ministerio de Educación) y sin cobro por escolarización. Su oferta educativa es a nivel preescolar, básico y medio y en 2010 tenía 329 alumnos. (iv) Liceo Aldea Educativa (LAE): Se inauguró el año 2006 en tanto sección de educación media de la enseñanza municipal. Dos características marcan su inicio, primero su instalación lejana de (a unos 4 kilómetros de Hanga Roa, único centro poblado de isla) tema relevante, ya que en la isla no existe movilización colectiva regular y, en segundo lugar, que fue parte de un proceso mayor de desarrollo cultural financiado con fondos nacionales e internacionales (UNESCO; MINEDUC; Ilustre Municipalidad de Isla de Pascua 2001). Es el colegio más pequeño de la isla (poco más de 120 alumnos) y el que más alumnos ha perdido en los últimos años. Tiene una sección de enseñanza científico humanista y dos de enseñanza técnico profesional (en turismo y especialidad agrícola). La clasificación socioeconómica que se hace de las familias es, al igual que el colegio anterior, de rango medio.

\section{Efecto de la Dinámica Competitiva del Actual Sistema Escolar en Isla de Pascua: Datos e Hipótesis}

El actual sistema escolar en Isla de Pascua, de tipo competitivo, ha generado algunas consecuencias observables como la disminución de la matrícula municipal, la consolidación de establecimientos relativamente pequeños en matrícula, resultados promedios disímiles en los rendimientos educativos (medidos por SIMCE) y una segmentación social del alumnado en los establecimientos. En términos sociales, culturales y políticos, los procesos de segmentación educacional constituyen el lado oscuro de todo proceso de ampliación de la diversidad de oferta educacional. Conceptualmente, la segmentación educacional se refiere a la distribución de alumnos en diferentes colegios de un territorio de acuerdo con características socioeconómicas, educativas y/o de otro tipo, lo que puede impactar en los aprendizajes de los alumnos y eventualmente en los procesos de cohesión social que se esperan del sistema escolar. La pregunta entonces respecto del sistema educacional de Isla de Pascua es si este presenta elementos de segmentación y si ella es de tipo socioeconómica, de capital cultural, étnica o educativa y en qué medida su existencia afecta los 
objetivos del propio sistema educacional, en términos de distribuir equitativamente los conocimientos entregados.

\section{La disminución de la matrícula municipal y el aumento de la privada}

La Figura 1 da clara cuenta de este proceso durante la década pasada. Se observa el descenso y aumento simultáneo de ambos tipos de matrícula. La correspondencia entre ambas permite afirmar que el aumento de la segunda es a expensas de la primera y no solo por la llegada de nueva población escolar que eventualmente se hubiese matriculado en la educación privada, sin impactar negativamente en la matrícula municipal. La Figura 2 da cuenta del notable aumento de la matrícula escolar en la isla durante la década pasada, lo que explica en buena parte el mercado educacional que se abrió para nuevos colegios.

Se observa (Figura 3) que la formación del sistema educativo actual en la isla tuvo un impacto en el tamaño de los establecimientos constituyéndose cuatro colegios de tamaño pequeño considerando el promedio nacional urbano al respecto (que es en torno a 600 alumnos en educación básica y 400 en educación media), siendo particularmente de pequeño tamaño el liceo municipal (127 alumnos).

\section{Diferenciación en los rendimientos educativos (SIMCE)}

La Tabla 1 cuenta del último resultado de la prueba SIMCE (año 2011) nivel segundo medio y en ella se aprecian las diferencias de logro en los establecimientos.

\section{Impacto en la segmentación social de la población en los colegios de la isla}

Respecto del tema de la segmentación social ella resulta evidente a partir del ya señalado diferente nivel socioeconómico de las familias de cada colegio, de la existencia de alumnos prioritarios en solo dos de ellos y de los disimiles resultados SIMCE entre los colegios. Esto permite afirmar que el sistema educativo actual en Isla de Pascua se encuentra segmentado en términos socioeconómicos y educativos. La pregunta que surge es si esta segmentación alcanza niveles étnicos (rapa nui/ continentales) y para eso usamos los datos de la encuesta aplicada a nivel de enseñanza media.

En la Tabla 2 se observa que a nivel de la enseñanza media en la isla el $78 \%$ de los alumnos son de ascendencia rapa nui. Estas distribuciones cambian entre los colegios, ya que el LAE tiene un $90 \%$ de población rapa nui, el CHEE un $76 \%$

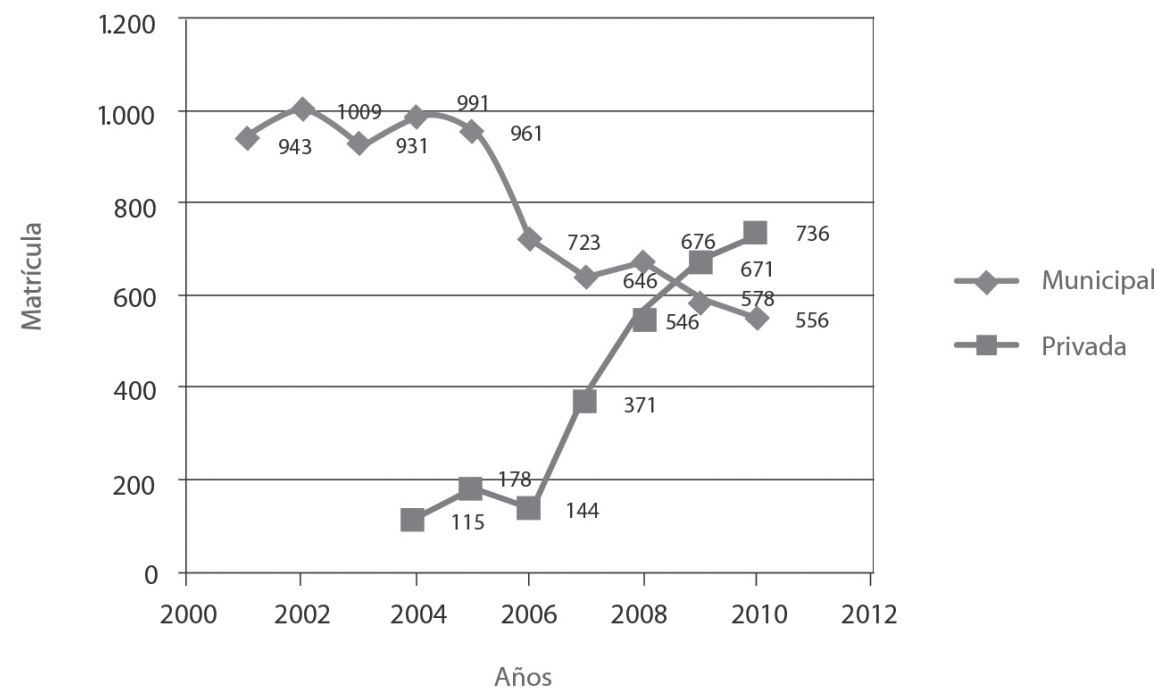

Figura 1. Matrícula escolar (básica y media) municipal y privada (particular subvencionada) en Isla de Pascua en el período 2001-2010. Elaboración propia a partir de datos del Ministerio de Educación.

Municipal and private (primary and secondary) school enrollment on Easter Island between 2001 and 2010.

Elaborated by the authors based on data from the Ministry of Education. 


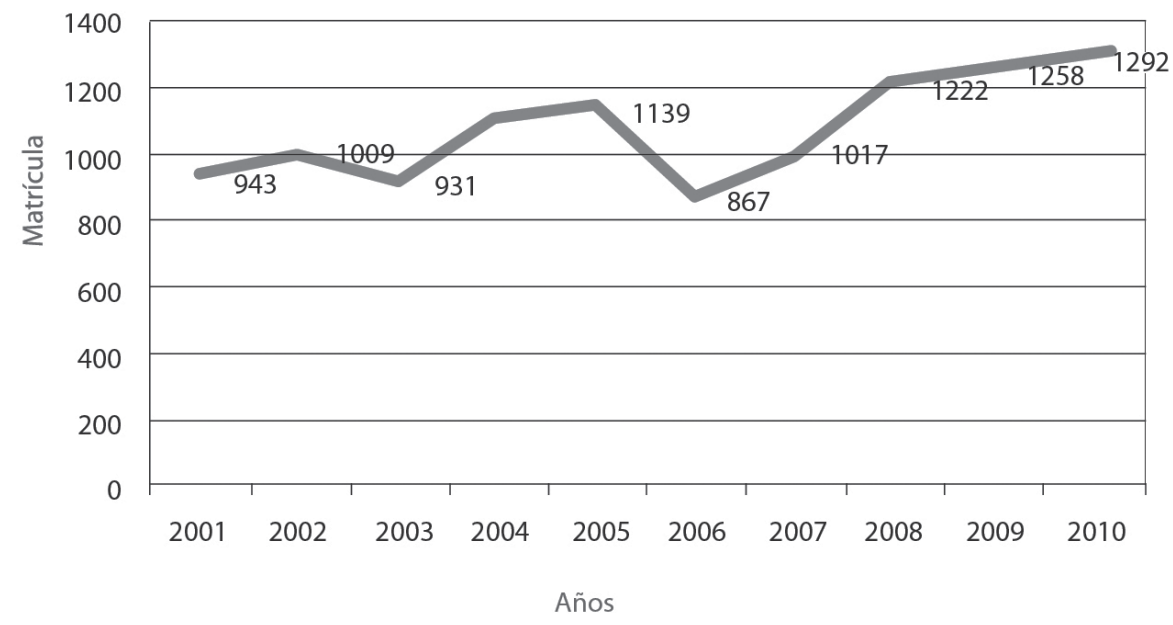

Figura 2. Evolución de la matrícula escolar (básica y media) en Isla de Pascua en el periodo 2001-2010. Elaboración propia a partir de datos del Ministerio de Educación.

Development of the primary and secondary school enrollment on Easter Island during 2001-2010. Elaborated by the authors using data from the Ministry of Education.

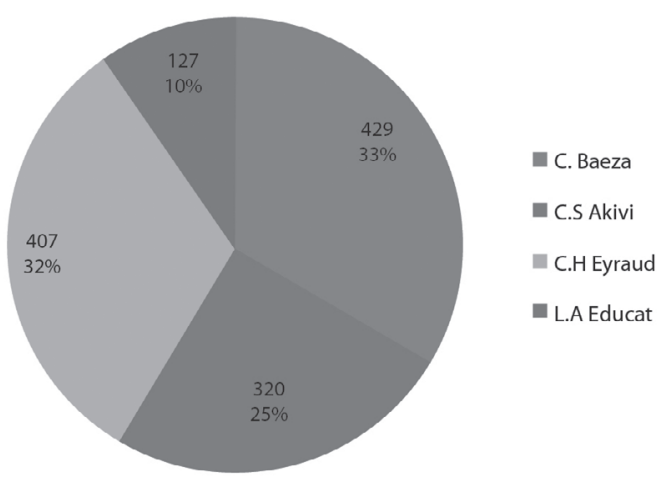

Figura 3. Matrícula escolar total y proporcional en los colegios de Isla de Pascua. Elaboración propia a partir de datos del Ministerio de Educación.

Total and proportional school enrollment on Easter Island schools. Elaborated by authors using data from the Ministry of Education. y el CSSA un 53\%. Se observa que en el caso de la ascendencia por padre y madre rapa nui a la vez es notablemente mayor en el caso del LAE que llega a un $38 \%$ de su alumnado en tal situación, la distribución total concentra los mayores porcentajes en la ascendencia solo materna o de ambos padres pero que esta última es más pronunciada en el LAE, es decir, se observan rasgos de distribución de alumnos y familias en los colegios que obedece a criterios de conformación étnica de las familias.

Por último interesa conocer el capital cultural de las familias rapa nui en cada colegio. Para ello construimos un índice simple (Tabla 3 ) de acuerdo con el nivel de escolaridad declarado respecto de los padres de los alumnos y promediando los valores respectivos de sus padres y madres. Esto lleva a la

Tabla 2. Distribución étnica porcentual de la matrícula por colegio en enseñanza media en Isla de Pascua. Percentage of ethnical distribution of secondary school enrollments per school on Easter Island.

\begin{tabular}{|c|c|c|c|c|c|}
\hline \multirow{2}{*}{$\begin{array}{c}\stackrel{\text { Porcentaje de alumnos }}{\longrightarrow} \\
\text { Establecimientos educacionales }\end{array}$} & \multicolumn{2}{|c|}{$\begin{array}{l}\text { Porcentaje de alumnos } \\
\text { según origen étnico }\end{array}$} & \multicolumn{3}{|c|}{$\begin{array}{l}\text { Porcentaje de alumnos rapa nui } \\
\text { desagregrado según filiación }\end{array}$} \\
\hline & $\begin{array}{l}\text { \% de alumnos } \\
\text { continentales de } \\
\text { enseñanza media }\end{array}$ & $\begin{array}{l}\% \text { de alumnos } \\
\text { rapa nui de } \\
\text { enseñanza media }\end{array}$ & $\begin{array}{l}\text { Alumnos con padre } \\
\text { rapa nui y madre } \\
\text { continental }\end{array}$ & $\begin{array}{c}\text { Alumnos con padre } \\
\text { continental y } \\
\text { madre rapa nui }\end{array}$ & $\begin{array}{c}\text { Alumnos con } \\
\text { padre y madre } \\
\text { rapa nui }\end{array}$ \\
\hline \multicolumn{6}{|l|}{$\downarrow$} \\
\hline Liceo Aldea Educativa & 10 & 90 & 23 & 35 & 42 \\
\hline Colegio San Sebastián de Akivi & 47 & 53 & 29 & 36 & 35 \\
\hline Colegio Hermano Eugenio Eyraud & 24 & 76 & 25 & 45 & 30 \\
\hline Total & 22 & 78 & 25 & 38 & 37 \\
\hline
\end{tabular}

Fuente: Trabajo de terreno encuesta a alumnos de enseñanza media en Isla de Pascua julio 2012. 
Tabla 3. Distribución de índices de capital cultural de los padres de los alumnos rapa nui en enseñanza media. Distribution of cultural capital indexes of the parents of high school Rapa Nui students.

\begin{tabular}{lrrrrr}
\hline $\begin{array}{l}\text { Índice de capital cultural de los } \rightarrow \\
\text { padres de los alumnos }\end{array}$ & Capital alto & Capital medio & Capital bajo & Total & $\begin{array}{c}\text { Índice } \\
\text { proporcional } \\
\text { comparado }^{2}\end{array}$ \\
Establecimiento $\downarrow$ & & & & & \\
\hline Liceo Aldea Educativa Rapa Nui & $56(48,7 \%)$ & $48(41,8 \%)$ & $11(9,5 \%)$ & $115(100 \%)$ & 69,5 \\
Colegio San Sebastián de Akivi & $12(44,5 \%)$ & $9(33,3 \%)$ & $6(22,2 \%)$ & $27(100 \%)$ & 61,0 \\
Colegio Hermano Eugenio Eyraud & $44(67,7 \%)$ & $19(29,2 \%)$ & $2(3,1 \%)$ & $65(100 \%)$ & 82,3 \\
\hline Total & $\mathbf{1 1 2}(\mathbf{5 4 , 1 \% )}$ & $\mathbf{7 6 ( 3 6 , 7 \% )}$ & $\mathbf{1 9 ( 9 , 2 \% )}$ & $\mathbf{2 0 7}(\mathbf{1 0 0 \%})$ & 72,5 \\
\hline
\end{tabular}

Fuente: Trabajo de terreno encuesta a alumnos de enseñanza media en Isla de Pascua, julio 2012.

${ }^{1}$ Índice construido sobre información de niveles educacionales del padre y madre del alumno y recodificado en los tres tramos señalados.

${ }^{2}$ Se refiere a que si todos los alumnos de un colegio pertenecieran a la categoría "capital alto" el valor sería 100 y si todos pertenecieran a la categoría "capital bajo" el valor sería 0 .

siguiente distribución de la población rapa nui en enseñanza media por colegio).

Se observa que el colegio CHEE tiene un índice comparado notablemente mayor que los otros dos colegios explicado en gran parte porque dos tercios de su alumnado se clasifica en "capital alto" y muy pocos en "capital bajo". En conclusión la población rapa nui que lleva sus hijos a la educación media está mayormente concentrada, en lo que a mayor capital cultural se refiere, en uno de los colegios.

\section{Conclusiones}

La escolarización en Isla de Pascua se desarrolló aceleradamente en comparación a lo que ocurrió en Chile continental, jugando un rol clave en la occidentalización y chilenización de la población rapa nui. En la actualidad, la estructura de su sistema educativo, con predominio de escuelas y matrícula privadas, coexiste con segmentaciones socioeconómicas y educativas entre los colegios de la isla. Dado que la mayor parte del alumnado actual en la isla pertenece al pueblo rapa nui y por lo tanto ellos están presentes en todos los colegios de la isla, es posible concluir un proceso de diferenciación intraétnica de tipo socioeconómico reflejado y refrendado en los colegios y que tiene su origen en cambios globales en la sociedad rapa nui, pero que es también asimilado por la estructura educativa actual. Cada uno de estos colegios tiene además características y resultados educativos específicos y solo uno de ellos tiene una educación con énfasis marcado en la lengua y cultura rapa nui. Los eventuales impactos de este fenómeno sobre los niveles de cohesión y de identidad en la sociedad rapa nui es algo que se debería evaluar en los años siguientes.

Agradecimientos: a Daniela Cienfuegos (profesora) y Sara Joiko (socióloga) por el apoyo en el análisis de la información recogida. A los directores de cada uno de los colegios de Isla de Pascua que tienen enseñanza media, por facilitar el acceso a la información y a los pares evaluadores de este artículo.

\section{Referencias Citadas}

Amunátegui, M. y V. Amunátegui 1856. De la Instrucción Primaria en Chile: lo que es, lo que Debería Ser. Imprenta del Ferrocarril, Santiago.

Annales de la Propagation de la Foi 1867. Tome trente-neuvième. Éditeur des Annales, Lyon.

Annales de la Propagation de la Foi 1869. Tome quarante-unième. Éditeur des Annales, Lyon.
Anderson, B. 2007. Comunidades Imaginadas. Reflexiones Sobre el Origen y la Difusión del Nacionalismo. Cuarta edición. Fondo de Cultura Económica, México.

Archer, M. 1984. Social Origins of Educational Systems. Sage, London.

Corvalán, J., G. Elacqua y F. Salazar 2010. El sector particular subvencionado en Chile. Tipologización y perspectivas frente a 
las nuevas regulaciones en MINEDUC Evidencias Para Políticas Públicas en Educación. MINEDUC, Santiago.

Cristino, C. 2010. Colonialismo y Neocolonialismo en Rapa Nui: una Reseña Histórica. En La Compañía Explotadora de Isla de Pascua. Patrimonio, Memoria e Identidad en Rapa Nui, editado por C. Cristino y M. Fuentes, pp. 19-52 Escaparate, Santiago.

Cristino, C., A. Recasens, P. Vargas, E. Edwards y L. González 1984. Isla de Pascua. Proceso, Alcances y Efectos de la Aculturación. Universidad de Chile, Santiago.

Comisión Verdad Histórica y Nuevo Trato con los Pueblos Indígenas 2008. Informe. Editado por el Comisionado Presidencial para Asuntos Indígenas Primera edición.

Durkheim, E. 1993 [1922]. Education et Sociologie. PUF, Paris.

Edwards, R. 1918. El Apóstol de Isla de Pascua José Eugenio Eyraud. Hermano de la Congregación de los Sagrados Corazones. Imprenta Chile, Santiago.

Estella, B. 1920. Los Misterios de Isla de Pascua. Imprenta Cervantes, Santiago.

Fisher, S. 2005. Island at the End of the World: The Turbulent History of Easter Island. Reaktion Books, London.

Giménez, G. 2006. El debate contemporáneo en torno al concepto de etnicidad. Cultura y Representaciones Sociales 1(1):129-144.

Gómez, R. 2010. Rapa nui and Chile, a Debate on Selfdetermination: A Notional and Legal Basis for the Political Decolonisation of Easter Island. Master Thesis in Pacific Studies, Victoria University of Wellington, Wellington.

González, S. 2002. Chilenizando a Tunupa. La Escuela Pública en el Tarapacá Andino. DIBAM, Santiago.

Makihara, M. 2005a. Rapa Nui ways of speaking Spanish: Language shift and socialization on Eastern Island. Language in Society 34:727-762.

----2005b. Being Rapa Nui, speaking Spanish: Children's voices on Easter Island. Anthropological Theory 5:117-134.

McCall, G. 1996. El Pasado en el Presente de Rapa Nui. En Etnografía. Sociedades Indígenas Contemporáneas y su Ideología, editado por J. Hidalgo, V. Schiapacasse, H.Niemeyer, C. Aldunate y P. Mege, pp. 17-46. Editorial Andrés Bello, Santiago.
Muñoz, D. 2007. Rapa nui Translocales. Reconfiguración de la Etnicidad Rapa nui en Santiago de Chile. Tesis para optar al Grado de Licenciado en Antropología. Universidad de Chile, Santiago.

PIIE 1984. Las transformaciones educacionales del régimen militar. Programa Interdisciplinario de Investigaciones en Educación, Santiago.

Ponce de León Atria, M. 2010. La llegada de la escuela y la llegada a la escuela. La extensión de la educación primaria en Chile (1840-1907). Historia 43:449-486.

Porteous, J.D.1981. The Modernization of Eastern Island. Western Geographical Series Volume 19. Department of Geography, University of Victoria, Victoria.

Ramírez, J.M. 2011. Historia de Rapa Nui. En Rapa Nui. Pasado, Presente, Futuro. UNESCO, pp. 11-32, UNESCO, Santiago.

Rojas, J. 2010. Historia de la Infancia en el Chile Republicano. JUNJI, Santiago.

Routledge, K. 1919. The Mystery of Eastern Island. The Story of an Expedition. Hazell, Watson and Viney, London.

Soto, C. y M. Fuente 2010. Vivir en tiempos de la compañía explotadora de Isla de Pascua. Una lectura al proceso desde el testimonio de los isleños. En La Compañía Explotadora de Isla de Pascua. Patrimonio, Memoria e Identidad en Rapa Nui, editado por C. Cristino y M. Fuentes pp. 255-276. Escaparate, Santiago.

Stambuk, P. 2010. Rongo. La Historia Oculta de Isla de Pascua. Pehuén, Santiago.

UNESCO, MINEDUC, Ilustre Municipalidad de Isla de Pascua 2001. Rapa Nui. Aldea Educativa. Plan Maestro. UNESCO, Santiago.

Zurob, C. 2009a. Ta'aku poki. Cambios y Continuidad en las Estrategias de Crianza Rapa Nui. Un Acercamiento a la Transmisión en Diferencia. Memoria para optar al Título de Antropóloga Social. Universidad de Chile, Santiago.

----2009b. El hogar estudiantil Hare Para Nui de Viña del Mar. Una Mirada a la educación, la Emigración y la diversidad en el contexto estatal. AIBR. Revista de Antropología Iberoamericana 4:84-112.

\section{Notas}

1 Nos referimos por fuerte o alta etnicidad a la existencia en un territorio delimitado de grupos humanos que se consideran cultural e históricamente distintos entre ellos o diferentes de la sociedad mayor que los circunda y a la que están vinculados en términos políticos y/o económicos. Respecto de este concepto de etnicidad ver Giménez 2006.

2 Es una evaluación regular y censal en Chile de aprendizaje de los alumnos (ver www.simce.cl).

3 El 90\% de los alumnos matriculados en los colegios ( $\mathrm{N}=270$ ) y dado que respondieron todos los alumnos presentes consideramos que esta encuesta es de tipo censal a nivel de la educación media (secundaria) en la isla. La encuesta fue aplicada en julio de 2012.
4 Benjamín Vicuña Mackenna señala: "Si de nuestro país ha partido la luz del evangelio y de la civilización a Pascua, debe ser también nuestra bandera la que allí flamee" citado en Estella 1920:92.

5 "Me parece que la República no puede dejar escapar esta ocasión de apropiarse muy legítimamente de un punto importante sobre sus costas... pero la utilidad más grande que puede Chile obtener de ella es el impedir... que una nación extranjera instale allí un barco a vapor..." Carta enviada del Obispo de Tahití al Reverendo Padre Superior de la Congregación de Valparaíso, el 6 de septiembre de 1871, referido en http://www.serindigena.org/libros_digitales/ cvhynt/v_i/1p/v1_pp_3_rapanui-_.html 
6 "Isla de Pascua, viene a ser un eslabón más del proceso expansionista chileno, siendo el único caso en que esta propagación se realizó por medio de un acuerdo y no por la militar" (Comisión Verdad Histórica y Nuevo Trato con los Pueblos Indígenas 2008:280).

7 "No podían movilizarse libremente por la isla, ni tampoco practicar labores de pesca sin solicitar las autorizaciones pertinentes en la Subdelegación" (Soto y Fuentes 2001:264).

8 Esta autora señala el relato de una antigua alumna rapa nui que vivió este proceso: "cuando no aprendíamos nosotros... levantaban la falda y con una varilla de ohe, con eso le daba en las piernas... pero es que no captábamos el castellano" (Zurob 2009a:97).

9 En 1986 según McCall (1996) los funcionarios de origen continental, junto a sus familias, llegaban al $20 \%$ de la población total de la isla.

10 La ley 16.441 del 1 de marzo de 1966 crea el Departamento de Isla de Pascua.

11 "Aquellas personas que hoy tienen entre 35 y 50 años aproximadamente corresponden a la segunda generación en diáspora... crecieron al alero de ver al continente como un lugar de oportunidades. Los padres de esta segunda generación coincidieron en ver que en el continente la educación era mejor que en la isla... que al contrario que ellos, había más posibilidades de llegar al continente" (Muñoz 2007:4).

12 La encuesta que aplicamos en julio de 2012 indicó que más del $80 \%$ de los alumnos expectativa de realizar estudios de educación superior una vez terminado el colegio. Este análisis también mostró que no existe diferencia significativa en estas expectativas entre los alumnos rapa nui y los continentales.

13 Se refiere a una categoría utilizada por la política educativa chilena y que en grueso alude a la condición de pobreza. Los niños clasificados como "prioritarios" son portadores de recursos adicionales al sostenedor de la escuela, con el fin de mejorar sus logros de aprendizaje.

14 Según el Ministerio de Educación esta categoría se refiere a ingresos familiares ubicados entre $\$ 280.001$ y $\$ 500.000$, a apoderados que tienen 11 y 12 años de escolaridad y a un $30,01 \%$ y un $55 \%$ de alumnos del colegio en condición de vulnerabilidad social. 